\title{
The effects of supplementation with P-Synephrine alone and in combination with caffeine on resistance exercise performance
}

Nicholas A. Ratamess ${ }^{1 *}$, Jill A. Bush', Jie Kang ${ }^{1}$, William J. Kraemer², Sidney J. Stohs ${ }^{3}$, Vincenzo G. Nocera', Megan D. Leise ${ }^{1}$, Keith B. Diamond ${ }^{1}$ and Avery D. Faigenbaum

\begin{abstract}
Background: Little is known concerning the potential ergogenic effects of $p$-synephrine supplementation. Therefore, the purpose of the present study was to examine the effects of supplementation with $p$-synephrine alone and in combination with caffeine on free-weight resistance exercise performance.

Methods: Twelve healthy, college-aged men performed a control (CT) resistance exercise protocol consisting of 6 sets of squats for up to 10 repetitions per set using $80 \%$ of their one repetition-maximum (1RM) with 2 min of rest in between sets. Each subject was randomly assigned (in double-blind, balanced manner) to a treatment sequence consisting of use of 3 supplements: $p$-synephrine ( $\mathrm{S} ; 100 \mathrm{mg}$ ), $p$-synephrine + caffeine (SCF; $100 \mathrm{mg}$ of $p$-synephrine plus $100 \mathrm{mg}$ of caffeine), or a placebo (P). For each supplement treatment (separated by 1 week), subjects consumed the supplement for 3 days prior to each protocol and the morning of each protocol, and subsequently did not consume any supplements for 3 days following (i.e. wash-out period). On each protocol day, subjects reported to the lab at a standard time, consumed a supplement, sat quietly for $45 \mathrm{~min}$, performed the resistance exercise protocol, and sat quietly for 30 min post exercise. Performance (repetition number, force, velocity and power), blood lactate, and ratings of perceived exertion (RPE) data were collected during each protocol.
\end{abstract}

Results: Supplements SCF and S produced a significantly $(P<0.05)$ greater number of repetitions performed than $C T$ (by $11.0 \pm 8.0 \%$ ) and P (by $6.0 \pm 7.0 \%$ ) and a $10.6 \pm 12.0 \%$ greater increase in volume load per protocol than CT and P. Most of the differences were seen during the last 3 sets. Mean power and velocity for all 6 sets were significantly higher in SCF compared to CT and P by $\sim 6.2 \pm 8.0 \%$. No supplement effects were observed in RPE or blood lactate, and no adverse side effects were observed or reported.

Conclusions: S and SCF augmented resistance exercise performance (total repetitions, volume load) without increasing blood lactate or RPE. The addition of caffeine in SCF increased mean power and velocity of squat performance. These results indicate supplementation with S and SCF can enhance local muscle endurance during resistance exercise.

\section{Background}

Thermogenic supplement use in various forms has increased in popularity among athletes and individuals targeting weight loss. These supplements target weight loss by increasing energy expenditure, the rate of fat oxidation, and by decreasing appetite [1-6]. Although their effects on anaerobic exercise performance have been equivocal [7-10], thermogenic supplements are thought

\footnotetext{
* Correspondence: ratamess@tcnj.edu

'Department of Health and Exercise Science, The College of New Jersey, Ewing, NJ 08628, USA

Full list of author information is available at the end of the article
}

to have mild ergogenic properties. Many thermogenic supplements contain compounds such as $p$-synephrine and caffeine [11]. $p$-Synephrine is the primary protoalkaloid derived from the immature fruits of Citrus aurantium (bitter orange, Seville orange). p-Synephrine also occurs in other orange-related species as Marrs sweet oranges, clementines and mandarin oranges $[12,13]$.

$p$-Synephrine has its hydroxyl group located in the para position on its benzene ring which has been shown to significantly alter its adrenergic receptor binding properties. It has low binding affinity for $\alpha-1$ and $\alpha-2$ as well as $\beta-1$ and $\beta-2$ adrenoreceptors [14]. Therefore, it exhibits 
little or no cardiovascular stimulation compared to ephedrine, $m$-synephrine (phenylephrine), and the catecholamines [14]. The ergogenic actions of $p$-synephrine are thought to be mediated mostly by $\beta-3$ adrenoreceptor activation leading to increased metabolic rate, lipolysis, and possibly reduced food intake $[14,15]$.

Stohs et al. [12] reviewed over 20 published and unpublished human studies involving $p$-synephrine with doses ranging from 5 to $80 \mathrm{mg}$ (alone and in combination with 132 to $704 \mathrm{mg}$ of caffeine). $p$-Synephrine alone or in combination with other nutrients increased resting metabolic rate and energy expenditure by up to $\sim 13 \%$, and led to modest reductions in body weight (all of the studies examining weight loss investigated $p$-synephrine in combination with other nutrients). However, potential ergogenic effects of $p$-synephrine are poorly understood particularly during resistance exercise.

Caffeine is a thermogenic methylxanthine alkaloid that acts as a central nervous system stimulant. It has been shown to increase energy expenditure and lipolysis and fat oxidation [16]. Caffeine is thought to enhance performance via several mechanisms including adenosine antagonism, potentiated cyclic AMP via phosphodiesterase inhibition, augmented muscle glycogen resynthesis, increased $\beta$ endorphin and catecholamine secretion, increased nerve conduction velocity and motor unit recruitment, reduced pain perception, and enhanced permeability, mobilization, and reduced uptake of intracellular calcium [16-22]. Some studies have shown caffeine consumption of $3-6 \mathrm{mg} / \mathrm{kg}$ of body mass increases endurance performance [16]. However, caffeine consumption during resistance exercise has produced equivocal findings. Some studies have shown ergogenic effects on muscle strength and endurance performance [23, 24], while others have failed to show performance augmentation [25, 26]. A meta-analysis has shown that caffeine consumption increases muscle strength and endurance but the effects may be musclegroup specific [27].

Given the paucity of studies examining $p$-synephrine supplement consumption during resistance exercise, the purpose of the present study was to examine resistance exercise performance concomitant with consumption of $p$-synephrine with or without a low dose of caffeine. It was our hypothesis that $p$-synephrine with and without caffeine would enhance repetition and power performance during resistance exercise.

\section{Methods}

\section{Experimental design}

In order to examine the primary hypotheses of the present study, a double-blinded, randomized, within-group crossover study design was used. After familiarization and preliminary baseline resistance exercise performance testing, subjects were randomly assigned (using a balanced design) to either a $p$-synephrine (100 mg; $\mathrm{S}), p$-synephrine + caffeine (100 mg $p$-synephrine plus $100 \mathrm{mg}$ caffeine; $\mathrm{SCF})$, or placebo (P) treatment. The resistance exercise protocol consisted of performing 6 sets of back squats for up to 10 repetitions per set using $80 \%$ of subjects' maximal strength with $2 \mathrm{~min}$ of rest in between sets at a standard time early in the morning in a fasted state. The protocol was performed 4 times (baseline and following consumption of each supplement). Subjects consumed each supplement in the form of chews for 3 days prior to and upon arrival at the laboratory the day of each protocol and then washed-out for 3 days following each protocol day. The 3-day pre-protocol supplementation period was utilized in order to expose subjects to the supplements on multiple occasions (to examine how subjects tolerated the supplemental doses) prior to performance assessment. Performance (repetition number, force, velocity, and power), blood lactate, and ratings of perceived exertion data were collected during each protocol.

\section{Subjects}

The subjects were healthy, college-aged (e.g., 2026 years) men $(N=12)$ who were former athletes with at least 2 years of resistance training experience from the student population at The College of New Jersey. Descriptive subject characteristics are shown in Table 1. None of the subjects were taking any medications, anabolic steroids, or nutritional supplements known to affect resistance exercise performance. All subjects had refrained from caffeine intake for at least 3 weeks prior to starting the study. Each subject was monitored during this time for symptoms such as headaches and fatigue in order to minimize potential confounding effects of caffeine withdrawal reversal. Three weeks was selected as withdrawal effects from low-to-moderate caffeine consumption typically subside within 10 days [28]. No withdrawal effects were reported upon initiation of prestudy familiarization and testing. Prior to this period, 5 subjects reported low daily caffeine intake $(<10-20 \mathrm{mg} /$ day), 3 subjects reported moderate caffeine intake (100-

Table 1 Subject descriptive characteristics

\begin{tabular}{lr}
\hline Variable & Mean \pm SD \\
\hline Age $(\mathrm{yrs})$ & $22.3 \pm 1.6$ \\
Height $(\mathrm{cm})$ & $179.5 \pm 7.8$ \\
Body mass $(\mathrm{kg})$ & $81.3 \pm 9.2$ \\
$\mathrm{RT}$ experience $(\mathrm{yrs})$ & $5.8 \pm 2.4$ \\
Percent body Fat $(\%)$ & $10.7 \pm 3.9$ \\
$\mathrm{VO}_{2}$ max $\left(\mathrm{ml} \cdot \mathrm{kg}^{-1} \cdot \mathrm{min}^{-1}\right)$ & $50.3 \pm 7.2$ \\
$1 \mathrm{RM}$ squat $(\mathrm{kg})$ & $135.6 \pm 24.4$ \\
$80 \%$ of $1 \mathrm{RM}$ squat $(\mathrm{kg})$ & $107.2 \pm 19.2$ \\
\hline
\end{tabular}

Key: $R T$ Resistance training, $\mathrm{VO}_{2}$ Oxygen consumption, $1 R M$ One repetition-maximum 
$200 \mathrm{mg} /$ day), and 4 subjects reported high daily caffeine intake (250-400 mg/day). Subjects were consistently questioned, completed diet records, and instructed not to consume known sources of caffeine during the experimental period. None of the subjects had any physiological or orthopedic limitations that could have affected lifting performance as determined by completion of a health history questionnaire prior to initiating the study. Subjects were instructed to refrain from exercise for 2 days prior to each protocol. This study was approved by The College of New Jersey's Institutional Review Board and each subject subsequently signed an informed consent document prior to participation.

\section{Preliminary screening and familiarization}

During the first visit to the Human Performance Laboratory, subjects completed a medical history questionnaire, informed consent document, were familiarized with the equipment and procedures, and were instructed on how to properly complete a 3-day dietary record. Height was measured using a wall-mounted stadiometer and body mass was measured using an electronic scale. $\mathrm{VO}_{2} \max$ was assessed using a progressive, multi-stage ramp protocol on a treadmill using a metabolic system (MedGraphics ULTIMA Metabolic System, MedGraphics Corporation, St. Paul, MN). Percent body fat was estimated via a three-site skinfold test. The sites measured were the pectoral, anterior thigh, and abdominal skinfolds [29]. The same research assistant performed all skinfold assessments. Body density was calculated using the equation of Jackson and Pollock [29] and percent body fat was calculated using the equation of Siri [30]. Dietary records were used to ensure subjects maintained normal kilocalorie and macronutrient intake throughout the study, and were completed for 3 days prior to the baseline protocol and for 3 days prior to each subsequent protocol. Each dietary record was analyzed using the Nutrition Calc Plus Version 3.4 software program (ESHA Research, Salem, OR). Total kilocalories, grams of fat, carbohydrates, and protein, and percent dietary intake of fats, carbohydrates, and protein were analyzed.

\section{Strength testing}

The one-repetition maximum (1RM) squat was used as a measure of strength using a standard protocol [31]. A warm-up set of 5-10 repetitions was performed using $40-60 \%$ of the perceived 1RM. After a 1-min rest interval, a set of 2-3 repetitions was performed at $60-80 \%$ of the perceived 1RM. Subsequently, 2-4 maximal trials were performed to determine the $1 \mathrm{RM}$ with $2-3 \mathrm{~min}$ rest intervals in between trials. A complete range of motion and proper technique was required for each successful 1RM trial. Subjects descended with the bar on the rear shoulders until their upper thighs were parallel to the ground. At that point a "lift" signal was given by a research assistant (to ensure proper depth) and the subject ascended to the starting position. Assessment of 1RM strength enabled calculation of the protocol loads (i.e. $80 \%$ of $1 \mathrm{RM}$ ).

\section{Control protocol}

A control (no supplement) resistance exercise protocol was performed first to establish baseline resistance exercise performance. Subjects reported to the laboratory early in the morning following a 10-h fast to minimize any confounding influence from prior meal consumption. Only water consumption was permitted. Upon arrival, each subject was encouraged to drink water ad libitum to pre-hydrate. Subjects had a cannula inserted into an antecubital vein for blood sampling. Subsequently, each subject was positioned on a reclining chair and sat quietly for 15 min prior to measurement of baseline blood lactate. Subjects then proceeded to sit quietly for an additional $45 \mathrm{~min}$ in a recumbent position. The quiet sitting period was used to mimic the time of supplement consumption during subsequent protocol sessions.

Subjects performed a standard warm-up consisting of 3 min of stationary cycling or walking, light stretching, and 2-3 light sets of squats with $30-65 \%$ of 1 RM. Water was provided ad libitum during this time. The resistance exercise protocol consisted of performing 6 sets of up to 10 repetitions (i.e. when momentary muscular failure was attained prior to the completion of 10 repetitions) of the free-weight back squat with $\sim 80 \%$ of 1 RM using 2-min rest intervals in between sets. Standard exercise technique was used and only those repetitions that met the criteria were counted. Resistance remained constant while total numbers of repetitions were recorded. Subjects used a self-selected cadence (with no rest in between repetitions) in order to maximize resistance exercise performance. Following each set, ratings of perceived exertion (RPE) were obtained using a category ratio (CR) 10-point (0-10) scale. Volume load (kg) was calculated as the number of completed repetitions $\mathrm{x}$ resistance used.

\section{Experimental protocol}

Following the control session, subjects were randomly assigned (in double-blind manner) to a balanced treatment sequence. The treatments involved use of 3 supplements: $p$-synephrine $(100 \mathrm{mg}), p$-synephrine + caffeine (100 mg of $p$-synephrine plus $100 \mathrm{mg}$ of caffeine), or a placebo treatment. Subjects consumed random treatment 1 for 3 consecutive days prior to returning to the laboratory. Subjects then reported to the laboratory the following morning in a fasted state similar to the control protocol session. After baseline measures and blood 
sampling, subjects consumed a dose of treatment 1 . They sat quietly for $45 \mathrm{~min}$ and subsequently initiated the resistance exercise protocol discussed previously. Each subject did not consume a supplement for the next 3 days (i.e. a 3-day "wash-out" period was used based on the half-lives and patterns of elimination of $p$-synephrine and caffeine) but then began supplementing with treatment 2 for 3 days prior to their next scheduled protocol session. Subjects arrived at the laboratory, consumed treatment 2, sat for $45 \mathrm{~min}$, and repeated the protocol described above. Following a 3-day wash-out period, subjects consumed treatment 3 for 3 days and repeated the protocol in a similar manner. Each supplement protocol session was identical to the control session with the exception of supplement consumption prior to the 45 min quiet sitting period (following baseline assessments). This cross-over design allowed each subject to experience all supplemental conditions in random sequence.

\section{Resistance exercise kinetics and kinematics}

Each resistance exercise protocol was performed on a portable force plate (Advanced Medical Technology Inc., Watertown, MA) with data collected at a frequency of $200 \mathrm{~Hz}$. Average and peak concentric ground reaction force per repetition were recorded and analyzed. Average bar velocity and power for the each repetition was measured with a Tendo ${ }^{\text {tw }}$ Power Output Unit (Tendo Sports Machines, Trencin, Slovak Republic). The Tendo ${ }^{\mathrm{Tm}}$ unit consists of a linear position transducer attached to the end of the barbell which measured linear displacement and time. Subsequently, average bar velocity and power were determined for each repetition. Power and velocity were averaged for each set (for all completed repetitions) and for each protocol. Test-retest reliability for the Tendo ${ }^{\mathrm{rm}}$ unit in our laboratory has consistently shown $R>0.90$ [32].

\section{Blood lactate measurements}

Subjects arrived at the laboratory in the early morning (at a standard time of day for all sessions) following an overnight fast. Venous blood samples were collected from subjects in a seated, semi-recumbent position at rest (T1), following the $45 \mathrm{~min}$ quiet sitting protocol (T2), immediately post-exercise (T3), and $15 \mathrm{~min}$ (T4), and $30 \mathrm{~min}$ (T5) post exercise. All blood samples were obtained using a 20-gauge Teflon cannula placed in a superficial forearm vein. The cannula was maintained patent via infusion of a heparin solution and blood was removed via a plastic syringe connected to a 3-way stopcock with a male luer lock adapter. T1 blood samples were drawn following a 15 min equilibration period. T3 blood samples were taken within $30 \mathrm{~s}$ of exercise cessation. Blood samples were collected into a Vacutainer ${ }^{\circ}$ tube containing $\mathrm{SST}^{\circ} \mathrm{Gel}$ and Clot Activator. An aliquot of each whole blood sample was removed and immediately used for determination of blood lactate. Whole blood lactate was analyzed in duplicate using an Analox GM7 enzymatic metabolite analyzer (Analox Instruments USA, Lunenburg, MA).

\section{Supplement composition and procedures}

The supplements used in the present study were in "chew" form (Advantra $Z^{\circ}$, Nutratech, Inc., West Caldwell, NJ). Each chew was identical in appearance, chocolate flavored, and identical in taste. All three supplements contained isomalt, maltitol syrup, cocoa powder, natural flavors, palm oil, soy lecithin, glycerin, and stevia. One serving consisted of two chews. Each serving of chews contained $30 \mathrm{kcal}$ from $8 \mathrm{~g}$ of carbohydrates. Thus, the placebo contained only these nutrients. The chews contained these nutrients with $100 \mathrm{mg}$ of $p$-synephrine from Citrus aurantium extract in two chews. The $p$-synephrine + caffeine chews consisted of the same nutrients plus $100 \mathrm{mg}$ of $p$ synephrine plus $100 \mathrm{mg}$ of caffeine. The supplements were administered to subjects in absolute doses. The rationale was to examine the supplement in a manner consistent with manufacturer's recommendations and to expand the $p$-synephrine literature base by providing a larger amount compared to previous studies which have mostly investigated absolute doses of 5-80 mg per day [12].

Subjects were instructed to consume each supplement for 3 days prior to arriving to the Human Performance Laboratory. Subjects were given the chews (6 in total) in bags labeled "A", "B", or "C", specific instructions on consumption, forms to complete by recording the time of day each supplement was consumed, and were instructed to return the empty wrappers as documentation of use. Two chews were consumed each day generally during the late morning, early afternoon hours. Both chews were consumed simultaneously and subjects were instructed to chew them completely and hold remnants under the tongue for at least 2 min prior to swallowing. Subjects were also given two chews during each resistance exercise protocol, i.e. prior to the 45 -min quiet sitting period resulting in consumption of 8 chews in total per treatment condition.

\section{Statistical analyses}

Descriptive statistics (means $\pm S D$ ) were calculated for all dependent variables. A 2-way (treatment $\mathrm{x}$ time point) analysis of variance (ANOVA) with repeated measures was used to analyze all within-subject data. Subsequent Tukey's post hoc tests were utilized to determine differences when significant main effects were obtained. A one-way ANOVA was used to analyze diet records and body weight changes. Pearson-product moment correlations were calculated for selected variables. For all 
statistical tests, a probability level of $P \leq 0.05$ denoted statistical significance.

\section{Results}

Dietary intake, body weight, and side effects

Statistical analysis of the 3-day dietary records showed that subject nutritional intake did not vary between the four experimental conditions. Subjects consumed an average of 2,594.2 $\pm 568.3 \mathrm{kcals}$ per day. Daily intake of protein, carbohydrates, and fat averaged $159.3 \pm 53.8,279.4 \pm$ 96.7 and $95.3 \pm 27.8 \mathrm{~g}$, respectively. This equated to $24.6 \pm$ $6.9,42.4 \pm 5.9$ and $32.3 \pm 5.4 \%$ of daily kilocaloric intake, respectively. Body mass did not significantly differ during each experimental protocol; $\mathrm{CT}=82.0 \pm 9.2 \mathrm{~kg} ; \mathrm{P}=81.7 \pm$ $9.4 \mathrm{~kg} ; \mathrm{S}=82.0 \pm 9.8 \mathrm{~kg}$; and $\mathrm{SCF}=81.6 \pm 9.4 \mathrm{~kg}$. Subjects reported no difference in taste between supplements as the chocolate flavor masked any potential bitter taste of the nutrients. In addition, no adverse side effects were reported throughout the experimental period.

\section{Repetition performance and volume load}

Table 2 presents repetition performance data. No supplement effect was observed when analyzing repetition number per set. However, significant differences were observed $[P=0.05]$ between treatments when total repetitions were analyzed. Supplements SCF and S produced significantly greater numbers of repetitions performed than CT and P. SCF and S produced $11.0 \pm 8.0 \%$ greater repetition numbers than the $\mathrm{CT}$ condition and $\sim 6.0 \pm 7.0 \%$ greater repetition numbers than the $\mathrm{P}$ treatment. In addition, a trend $(P=0.06)$ was observed where most of the differences shown between supplement conditions occurred between sets 4 and 6. A significant difference was observed $[P=0.03]$ between treatments. Supplements SCF $(4,609.1 \pm 1,175.2 \mathrm{~kg})$ and S $(4,623.0 \pm$ $1,117.5 \mathrm{~kg}$ ) produced a significantly greater volume load per protocol than $\mathrm{CT}(4,128.0 \pm 1,258.1 \mathrm{~kg})$ and $\mathrm{P}$
$(4,321.6 \pm 1,136 \mathrm{~kg})$. A significant main effect was observed $[P<0.0001]$ where repetition performance decreased with each successive set during all treatments. Specific differences are shown in Table 2.

\section{Ratings of Perceived Exertion (RPE)}

Table 3 presents RPE data following each set of resistance exercise. No treatment effects were observed over all 6 sets and no significant treatments effects were observed for the mean session RPE $[P=0.18]$. RPE obtained during each set was significantly $[P<0.001]$ negatively correlated with repetitions completed $(r=-0.48$ to $-0.67)$. A significant time effect was observed between the 1 st and 6th set of resistance exercise $[P<0.0001]$ where RPE values increased with each successive set.

\section{Average and peak power}

Average power data are presented in Table 4. No supplement effect was observed with regard to the pattern of reduction during each set. The mean power for all 6 sets was significantly higher in SCF compared to CT and $\mathrm{P}$ by $\sim 6.2 \pm 8.0 \%[P=0.03]$. Although mean power during $\mathrm{S}$ was higher than $\mathrm{CT}$ and $\mathrm{P}$, this value did not reach statistical significance. Average power was significantly negatively correlated to blood lactate values at T3 $(r=-0.29$ to -0.48$)$. A significant time effect was observed $[P<0.0001]$ where average power decreased with each successive set. No supplement effects were observed with regard to the pattern of peak power reduction during each set. No significant differences were observed $[P=0.67]$ between treatments when examining the mean peak powers of all 6 sets $(C T=1315.2 \pm$ $195.9 \mathrm{~W} ; \mathrm{P}=1289.8 \pm 170.6 \mathrm{~W} ; \mathrm{S}=1294.0 \pm 174.4 \mathrm{~W}$; $\mathrm{SCF}=1329.1 \pm 197.2 \mathrm{~W})$. A significant time effect was observed $[P<0.0001]$ where peak powers decreased with each successive set (data not shown).

Table 2 Repetition performance data

\begin{tabular}{lllll}
\hline & CT & P & S & SCF \\
\hline Set 1 & $10.0 \pm 0.0$ & $10.0 \pm 0.0$ & $10.0 \pm 0.0$ & $10.0 \pm 0.0$ \\
Set 2 & $8.1 \pm 2.3^{\mathrm{a}}$ & $9.2 \pm 1.1$ & $9.3 \pm 1.2$ & $8.9 \pm 1.7$ \\
Set 3 & $6.6 \pm 2.5^{\mathrm{ab}}$ & $6.7 \pm 3.0^{\mathrm{ab}}$ & $7.8 \pm 3.2^{\mathrm{ab}}$ & $7.7 \pm 2.3^{\mathrm{ab}}$ \\
Set 4 & $6.0 \pm 2.7^{\mathrm{abc}}$ & $5.9 \pm 3.1^{\mathrm{ab}}$ & $6.8 \pm 3.1^{\mathrm{ab}}$ & $6.5 \pm 2.8^{\mathrm{abc}}$ \\
Set 5 & $4.8 \pm 3.0^{\mathrm{abcd}}$ & $5.4 \pm 3.4^{\mathrm{abc}}$ & $5.4 \pm 2.8^{\mathrm{abcd}}$ & $5.7 \pm 3.1^{\mathrm{abcd}}$ \\
Set 6 & $4.0 \pm 2.7^{\mathrm{abcd}}$ & $4.1 \pm 2.5^{\mathrm{abcde}}$ & $4.9 \pm 3.0^{\mathrm{abcd}}$ & $5.3 \pm 3.4^{\mathrm{abcd}}$ \\
Reps sets 1 - 3 & $24.7 \pm 4.3$ & $25.9 \pm 3.6$ & $27.1 \pm 3.8$ & $26.6 \pm 3.7$ \\
Reps sets 4-6 & $14.8 \pm 8.1$ & $15.4 \pm 8.7$ & $17.1 \pm 8.5^{\#}$ & $17.5 \pm 9.2^{\#}$ \\
Total reps & $39.5 \pm 12.1$ & $41.3 \pm 11.9$ & $44.2 \pm 11.7^{*}$ & $44.1 \pm 12.4^{*}$ \\
\hline
\end{tabular}

Values are mean \pm SD

Key: $C T$ Control protocol, $P$ Placebo treatment, $S p$-synephrine treatment, SCF $p$-synephrine plus caffeine treatment

${ }^{a}-P \leq 0.05$ from set $1 ;{ }^{b}-P \leq 0.05$ from set $2 ;{ }^{c}-P \leq 0.05$ from set $3 ;^{d}-P \leq 0.05$ from set $4 ;{ }^{e}-P \leq 0.05$ from set $5 ;{ }^{*}-P \leq 0.05$ from $C T$ and $P ;{ }^{\#}-P=0.06$ from $C T$ and $P$ 
Table 3 Ratings of perceived exertion

\begin{tabular}{lllll}
\hline & $C T$ & $P$ & $S$ & SCF \\
\hline Set 1 & $5.36 \pm 1.5$ & $5.18 \pm 1.60$ & $5.14 \pm 1.27$ & $5.41 \pm 1.46$ \\
Set 2 & $6.55 \pm 1.6^{\mathrm{a}}$ & $6.55 \pm 2.02^{\mathrm{a}}$ & $6.23 \pm 1.57^{\mathrm{a}}$ & $6.36 \pm 1.63^{\mathrm{a}}$ \\
Set 3 & $7.14 \pm 1.10^{\mathrm{ab}}$ & $7.05 \pm 1.98^{\mathrm{ab}}$ & $6.73 \pm 1.79^{\mathrm{ab}}$ & $7.00 \pm 1.84^{\mathrm{ab}}$ \\
Set 4 & $7.55 \pm 1.29^{\mathrm{abc}}$ & $7.59 \pm 1.71^{\mathrm{abc}}$ & $7.18 \pm 1.94^{\mathrm{abc}}$ & $7.50 \pm 1.72^{\mathrm{abc}}$ \\
Set 5 & $7.91 \pm 1.22^{\mathrm{abc}}$ & $7.91 \pm 1.51^{\mathrm{abc}}$ & $7.50 \pm 1.66^{\mathrm{abc}}$ & $7.73 \pm 1.68^{\mathrm{abc}}$ \\
Set 6 & $8.91 \pm 1.22^{\mathrm{abcde}}$ & $8.55 \pm 1.37^{\mathrm{abcde}}$ & $8.27 \pm 1.49^{\mathrm{abcde}}$ & $8.36 \pm 1.36^{\mathrm{abcde}}$ \\
Mean & $7.23 \pm 1.12$ & $7.14 \pm 1.58$ & $6.84 \pm 1.49$ & $7.06 \pm 1.44$ \\
\hline
\end{tabular}

Values are mean \pm SD

Key: $C T$ Control protocol, $P$ Placebo treatment, $S p$-synephrine treatment, SCF $p$-synephrine plus caffeine treatment

${ }^{a}-P \leq 0.05$ from set $1 ;{ }^{b}-P \leq 0.05$ from set $2 ;^{c}-P \leq 0.05$ from set $3 ;^{d}-P \leq 0.05$ from set $4 ;^{e}-P \leq 0.05$ from set 5

\section{Average and peak velocity $(\mathrm{m} / \mathrm{s})$}

Average velocity data are presented in Table 5. No supplement effect was observed with regard to the pattern of reduction during each set. The mean velocity per set was significantly greater $[P=0.04]$ in SCF than $\mathrm{CT}$ and $\mathrm{P}$ by $\sim 6.5 \pm 8.0 \%$. Although mean velocity during $\mathrm{S}$ was higher than $\mathrm{CT}$ and $\mathrm{P}$, this value did not reach statistical significance. A significant time effect was observed $[P<0.0001]$ where average velocity decreased with each successive set. No supplement effect was observed with regard to the pattern of peak velocity reduction during each set. No significant differences were observed $[P=0.84]$ between treatments when examining the mean peak velocities of all 6 sets $\left(\mathrm{CT}=0.71 \pm 0.06 \mathrm{~m} \cdot \mathrm{s}^{-1} ; \mathrm{P}=0.70 \pm 0.10 \mathrm{~m} \cdot \mathrm{s}^{-1} ; \mathrm{S}=0.70\right.$ $\left.\pm 0.07 \mathrm{~m} \cdot \mathrm{s}^{-1} ; \mathrm{SCF}=0.72 \pm 0.08 \mathrm{~m} \cdot \mathrm{s}^{-1}\right)$. A significant time effect was observed $[P<0.0001]$ where peak velocities decreased with each successive set (data not shown).

\section{Peak and average concentric force $(\mathrm{N})$}

Peak concentric force data are presented in Table 6. No supplement effect was observed with regard to the pattern of peak force reduction during each set. No significant differences were observed $[P=0.21]$ between treatments when examining the mean peak concentric force of all 6 sets. Although S and SCF yielded higher peak concentric force (up to $\sim 2.5 \%$ ) than $\mathrm{CT}$ and $\mathrm{P}$, respectively, these differences did not reach statistical significance. A significant time effect was observed $[P<0.0001]$ where peak concentric force decreased with each successive set. No supplement effect was observed with regard to the pattern of average force reduction during each set. The mean concentric force across all 6 sets did not significantly differ among conditions $(\mathrm{CT}=1875.9 \pm 229.6 \mathrm{~N} ; \mathrm{P}=1901.1$ $\pm 210.5 \mathrm{~N} ; \quad \mathrm{S}=1898.1 \pm 217.0 \quad \mathrm{~N} ; \quad \mathrm{SCF}=1902.7 \pm$ $222.9 \mathrm{~N})[P=0.24]$. A significant time effect was observed $[P=0.03]$ where average concentric force decreased from set 1 through set 6 at various points (data not shown).

\section{Blood lactate}

Blood lactate data are shown in Fig. 1. No significant supplement effects were observed $[P=0.98]$. The lactate responses in $\mathrm{S}$ and SCF were similar to $\mathrm{CT}$ and P despite a greater volume load and repetition number performed during each protocol. A significant time effect was observed $[P<0.0001]$ where blood lactate was significantly elevated at T3, T4, and T5 compared to T1 and T2 with T3 showing the highest values immediately post resistance exercise.

Table 4 Average power data (Watts)

\begin{tabular}{lllll}
\hline & $C T$ & $P$ & $S$ & SCF \\
\hline Set 1 & $827.7 \pm 160.8$ & $870.4 \pm 153.9$ & $860.8 \pm 155.0$ & $875.6 \pm 160.4$ \\
Set 2 & $734.3 \pm 127.2^{\mathrm{a}}$ & $756.7 \pm 144.3^{\mathrm{a}}$ & $777.5 \pm 160.9^{\mathrm{a}}$ & $794.4 \pm 152.5^{\mathrm{a}}$ \\
Set 3 & $681.2 \pm 126.6^{\mathrm{ab}}$ & $677.1 \pm 119.2^{\mathrm{ab}}$ & $703.6 \pm 186.4^{\mathrm{ab}}$ & $729.7 \pm 150.5^{\mathrm{ab}}$ \\
Set 4 & $653.1 \pm 134.1^{\mathrm{ab}}$ & $622.6 \pm 133.7^{\mathrm{abc}}$ & $642.8 \pm 169.4^{\mathrm{abc}}$ & $682.4 \pm 144.6^{\mathrm{abc}}$ \\
Set 5 & $600.9 \pm 113.8^{\mathrm{abcd}}$ & $598.2 \pm 131.2^{\mathrm{abc}}$ & $622.8 \pm 140.2^{\mathrm{abc}}$ & $644.7 \pm 153.0^{\mathrm{abcd}}$ \\
Set 6 & $595.1 \pm 100.5^{\mathrm{abcd}}$ & $574.4 \pm 114.6^{\mathrm{abcde}}$ & $561.4 \pm 121.4^{\mathrm{abcde}}$ & $614.8 \pm 141.8^{\mathrm{abcde}}$ \\
Mean & $697 \pm 108.6$ & $697 \pm 106.5$ & $718.8 \pm 124.6$ & $743.0 \pm 129.3^{*}$ \\
\hline
\end{tabular}

Values are mean \pm SD

Key: CT Control protocol, $P$ Placebo treatment, $S p$-synephrine treatment, SCF $p$-synephrine plus caffeine treatment

${ }^{a}-P \leq 0.05$ from set $1 ;{ }^{b}-P \leq 0.05$ from set $2 ;^{c}-P \leq 0.05$ from set $3 ;^{d}-P \leq 0.05$ from set $4 ;^{e}-P \leq 0.05$ from set $5 ;^{*}-P \leq 0.05$ compared to $C T$ and $P$ 
Table 5 Average velocity data $\left(\mathrm{m} \cdot \mathrm{s}^{-1}\right)$

\begin{tabular}{lllll}
\hline & $C T$ & $P$ & $S$ & SCF \\
\hline Set 1 & $0.46 \pm 0.06$ & $0.49 \pm 0.06$ & $0.47 \pm 0.06$ & $0.48 \pm 0.05$ \\
Set 2 & $0.41 \pm 0.05^{\mathrm{a}}$ & $0.42 \pm 0.07^{\mathrm{a}}$ & $0.43 \pm 0.07^{\mathrm{a}}$ & $0.44 \pm 0.07^{\mathrm{a}}$ \\
Set 3 & $0.38 \pm 0.06^{\mathrm{ab}}$ & $0.38 \pm 0.07^{\mathrm{ab}}$ & $0.38 \pm 0.09^{\mathrm{ab}}$ & $0.40 \pm 0.07^{\mathrm{ab}}$ \\
Set 4 & $0.36 \pm 0.06^{\mathrm{ab}}$ & $0.34 \pm 0.07^{\mathrm{abc}}$ & $0.35 \pm 0.09^{\mathrm{abc}}$ & $0.38 \pm 0.08^{\mathrm{abc}}$ \\
Set 5 & $0.33 \pm 0.06^{\mathrm{abcd}}$ & $0.33 \pm 0.08^{\mathrm{abc}}$ & $0.34 \pm 0.07^{\mathrm{abc}}$ & $0.35 \pm 0.08^{\mathrm{abcd}}$ \\
Set 6 & $0.33 \pm 0.06^{\mathrm{abcd}}$ & $0.32 \pm 0.07^{\mathrm{abcde}}$ & $0.31 \pm 0.07^{\mathrm{abcde}}$ & $0.34 \pm 0.08^{\mathrm{abcde}}$ \\
Mean & $0.38 \pm 0.05$ & $0.38 \pm 0.06$ & $0.39 \pm 0.07$ & $0.40 \pm 0.07^{*}$ \\
\hline
\end{tabular}

Values are mean \pm SD

Key: CT Control protocol, $P$ Placebo treatment, $S p$-synephrine treatment, SCF $p$-synephrine plus caffeine treatment

${ }^{a}-P \leq 0.05$ from set $1 ;^{b}-P \leq 0.05$ from set $2 ;^{c}-P \leq 0.05$ from set $3 ;^{d}-P \leq$ 0.05 from set $4 ;{ }^{e}-P \leq 0.05$ from set $5 ;^{*}-P \leq 0.05$ compared to $C T$ and $P$

\section{Discussion}

The unique finding of the present study was that supplementation with $100 \mathrm{mg}$ of $p$-synephrine alone and in combination with $100 \mathrm{mg}$ of caffeine significantly augmented resistance exercise performance compared to $\mathrm{P}$ and CT treatments. The SCF and S treatments produced significantly greater numbers of repetitions performed than CT and P and a $10.6 \pm 12.0 \%$ greater increase in volume load per protocol than $\mathrm{CT}$ and P. Mean power and velocity for all 6 sets were significantly higher in SCF compared to CT and P. No supplement effects were observed in RPE or blood lactate and no side effects were reported throughout the experimental period.

The results of the present study showed ergogenic effects of S and SCF supplementation for increasing repetition performance and volume load during resistance exercise. Both S and SCF treatments led to a nearly 3repetition augmented performance across all sets although most of the enhancement was seen during the last 3-4 sets. To our knowledge, this is first study to demonstrate an ergogenic potential of $p$-synephrine supplementation when consumed solely or in combination with caffeine during resistance exercise.

The mechanism(s) leading to the enhanced performance remains unknown. The thermogenic actions of $p$ - synephrine are thought to be mediated mostly by $\beta-3$ adrenoceptor activation predominantly in adipose tissue with weak binding affinity to $\beta-1$ and $\beta$-2-adrenoceptors $[14,15]$. Other studies have shown $p$-synephrine can increase liver glycogenolysis [33] and increase skeletal muscle glucose uptake [34]. The extent to which these lipolytic and metabolic effects of $p$-synephrine augment resistance exercise performance remains unknown and may depend upon how much substrate depletion serves as a performance limiting factor (i.e. for 6 sets of barbell squats in the present study). Recent evidence has shown skeletal muscle possesses $\beta-3$ adrenoceptors $[35,36]$ although specific activation pathways influencing muscle strength, power, and endurance remain speculative. Murphy et al. [35] studied the effects of the $\beta-3$ agonist BRL 37344 on rat soleus muscles and reported increased $\mathrm{Na}+/ \mathrm{K}+$ ATPase pump activity that was mediated through $\beta-2$ adrenoceptors. BRL 37344 facilitated intracellular $\mathrm{Na}+$ removal and induced rapid force recovery after rat soleus muscles were fatigued by $16 \%$ following incubation with potassium. Using a more selective $\beta-3$ agonist CL 316,243, Miniaci et al. [36] reported increased skeletal muscle protein synthesis via the PI3K-mTOR-p70(S6k) signaling pathway. Taken together, there is growing evidence that $\beta$ 3 receptor agonists may influence skeletal muscle contractility either directly or indirectly through $\beta-2$ adrenoceptor activation. Although the mechanism(s) remains to be elucidated, the effects of $p$-synephrine on skeletal muscle performance via either $\beta-3$ adrenoceptor stimulation, possible cell signaling from other $\beta$ adrenoceptors, or CNS activation requires further study.

Interestingly, the addition of $100 \mathrm{mg}$ of caffeine to $100 \mathrm{mg} p$-synephrine did not augment repetition performance and volume load. Similar findings were reported where the addition of caffeine failed to augment the effects of ephedrine [37, 38]. Caffeine, by itself and in combination with other nutrients, has been shown to be ergogenic for various components of sports performance, aerobic endurance, and anaerobic power $[16,18,20]$. Various studies have shown caffeine enhances resistance

Table 6 Peak concentric force data (N)

\begin{tabular}{lllll}
\hline & CT & $P$ & $S$ & SCF \\
\hline Set 1 & $2256.5 \pm 290.3$ & $2295.1 \pm 281.6$ & $2287.8 \pm 286.2$ & $2328.8 \pm 306.5$ \\
Set 2 & $2232.6 \pm 276.9$ & $2262.0 \pm 296.4^{\mathrm{a}}$ & $2265.9 \pm 283.2$ & $2301.0 \pm 289.7$ \\
Set 3 & $2217.4 \pm 249.5$ & $2249.4 \pm 272.0^{\mathrm{a}}$ & $2252.7 \pm 259.0^{\mathrm{a}}$ & $2277.1 \pm 283.6^{\mathrm{ab}}$ \\
Set 4 & $2207.0 \pm 254.8^{\mathrm{a}}$ & $2224.5 \pm 270.8^{\mathrm{a}}$ & $2251.9 \pm 280.7^{\mathrm{a}}$ & $2262.0 \pm 287.0^{\mathrm{ab}}$ \\
Set 5 & $2184.1 \pm 258.4^{\mathrm{ab}}$ & $2208.4 \pm 251.9^{\mathrm{abc}}$ & $2224.9 \pm 267.8^{\mathrm{ab}}$ & $2227.5 \pm 268.6^{\mathrm{abcd}}$ \\
Set 6 & $2175.3 \pm 252.9^{\mathrm{ab}}$ & $2170.9 \pm 240.4^{\mathrm{abcde}}$ & $2209.7 \pm 244.0^{\mathrm{abc}}$ & $2214.3 \pm 241.6^{\mathrm{abcd}}$ \\
Mean & $2212.1 \pm 258.0$ & $2235.0 \pm 266.7$ & $2248.8 \pm 266.9$ & $2268.5 \pm 277.1$ \\
\hline
\end{tabular}

Values are mean \pm SD

Key: CT Control protocol, $P$ Placebo treatment, $S p$-synephrine treatment, SCF $p$-synephrine plus caffeine treatment

${ }^{a}-P \leq 0.05$ from set $1 ;{ }^{b}-P \leq 0.05$ from set $2 ;{ }^{c}-P \leq 0.05$ from set $3 ;^{d}-P \leq 0.05$ from set $4 ;^{e}-P \leq 0.05$ from set 5 


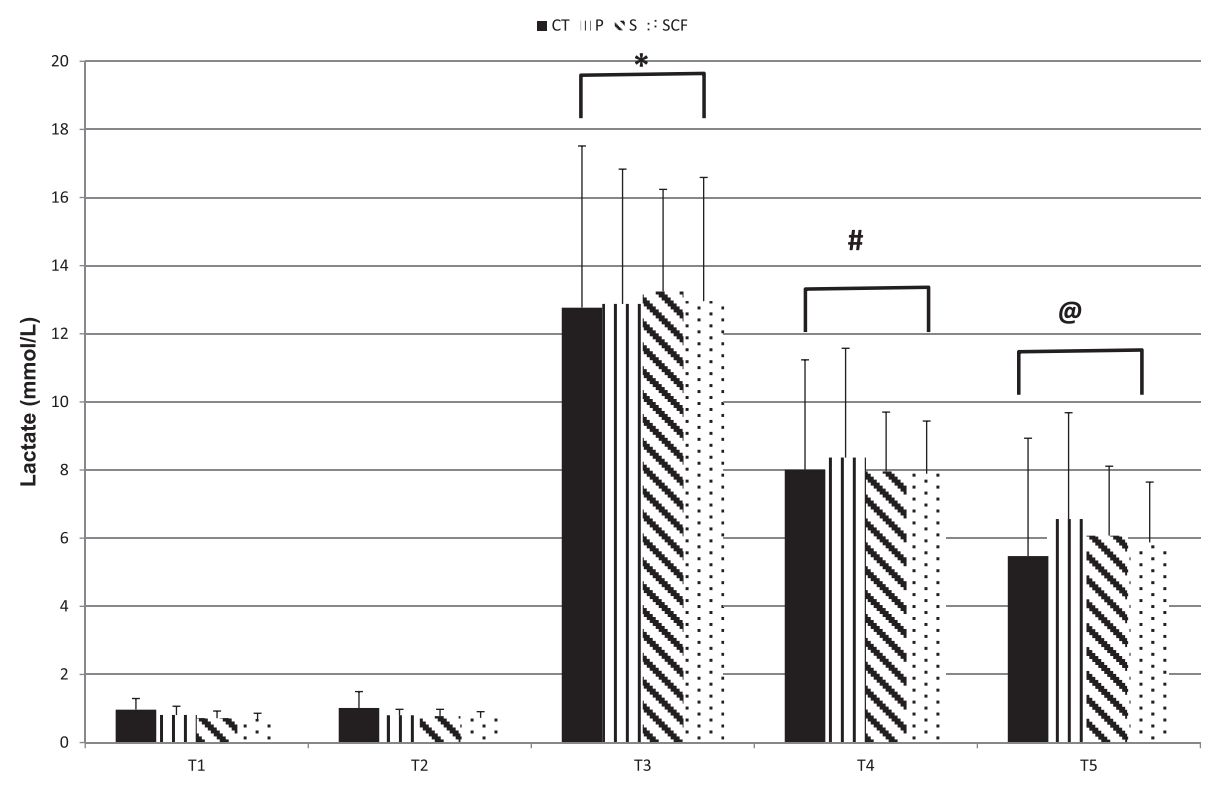

Fig. 1 Blood Lactate Response. Key: CT Control protocol, $P$ Placebo treatment, S p-synephrine treatment, SCF $p$-synephrine plus caffeine treatment; * $-P \leq 0.05$ from T1, T2, T4, and T5; \# - $P \leq 0.05$ from T1, T2, T3, and T5; @ - $P \leq 0.05$ from T1, T2, T3, and T4. No differences were observed in between treatments. Data presented are means \pm SD

exercise performance [18], isometric and isokinetic peak torque and power $[19,21,23,39]$, the number of repetitions performed to failure [24, 40-42] and maximal strength [43], and attenuates reductions in maximal strength and power seen during morning hours [44]. However, some studies have shown caffeine consumption did not augment peak torque [45], 1RM strength [25, 43, 46], or maximal repetition performance/volume load for at least some exercises assessed [38, 43, 46].

Trained subjects may be more responsive to caffeine intake $[18,21,47]$ and the likelihood of improvement increases with large muscle group size and location $[18,23,27,42,48]$. In a meta-analysis, Warren et al. [27] reported that caffeine exhibited some level of ergogenic improvements in 23 of 27 studies [augmented maximal voluntary contractile (MVC) strength of $\sim 4 \%$ and local muscle endurance by $14 \%$. Astorino \& Roberson [47] reviewed 11 studies and concluded that caffeine was more likely to increase resistance exercise repetition number (mean improvement of approximately $9.4 \%$ ) than 1 RM strength.

The vehicle of consumption and dose of caffeine affects the acute response. Most studies demonstrating ergogenic effects of caffeine utilized anhydrous caffeine consumption at moderate-to-high doses typically ranging from 2 to $9 \mathrm{mg} / \mathrm{kg}$ of body mass [27, 47]. Astorino et al. [49] reported only a higher dose of caffeine (5 versus $2 \mathrm{mg} / \mathrm{kg}$ ) improved isokinetic resistance exercise performance by $5-8 \%$ thereby demonstrating a doseresponse relationship. In the present study caffeine intake was low and given in a standard absolute amount within each chew averaging $1.23 \mathrm{mg} / \mathrm{kg}$ of body mass. Thus, it is possible that a higher dose of caffeine is needed to augment repetition performance and volume load. However, the potentiating effect of $p$-synephrine consumed in combination with caffeine needs to be considered. $p$-Synephrine alone augmented resistance exercise performance similarly to the SCF treatment. The amount of $p$-synephrine consumed in the present study was higher than most studies [11] but similar to one study [13] although these studies did not examine resistance exercise performance. Our data indicate that the addition of only $100 \mathrm{mg}$ of caffeine to the chews may not be sufficient to augment further increases in local muscle endurance.

The addition of caffeine to $p$-synephrine did augment repetition average velocity and power. Average power and velocity for all 6 sets were significantly higher in SCF compared to CT and P by $\sim 6.2 \pm 8.0 \%$. Although mean power during $\mathrm{S}$ was higher than $\mathrm{CT}$ and $\mathrm{P}$ (by $\sim 3 \%$ ), these values did not reach statistical significance. The mechanisms of caffeine's ergogenic effects are vast and difficult to isolate during exercise. Caffeine is a central nervous system stimulant that increases lipolysis, potentiates cyclic AMP via phosphodiesterase inhibition, and augments post-exercise muscle glycogen storage [20]. However, during high-intensity resistance exercise other mechanisms appear more plausible. Caffeine may increase contractile force through mobilization of intracellular calcium, increased calcium release from the sarcoplasmic reticulum, and decreased calcium uptake [20]. Other resistance exercise studies have shown 
increased muscle fiber conduction velocity [19] and EMG activity [39] following caffeine consumption possibly indicative of greater motor unit recruitment [27]. Adenosine antagonism, reduced pain perception, and blunted perceived exertion during high-intensity exercise performance may also play pivotal roles [18, 24, 40]. Thus, it appears multiple mechanisms in combination could contribute to caffeine's ergogenic effects of average velocity and power observed during each set.

An interesting finding was that the addition of $100 \mathrm{mg}$ of caffeine to $100 \mathrm{mg}$ of $p$-synephrine augmented average power during each set despite similar total repetitions and volume load performed between $\mathrm{S}$ and SCF treatments. Further analysis showed that the average and peak power obtained during each repetition that preceded muscular failure was still greater in SCF. This indicates that the subjects performed more powerful repetitions up until the point of momentary muscular exhaustion. However, the enhanced power did not result in a greater number of repetitions or volume load performed in SCF compared to S. In fact, no significant correlations were shown between average or peak power values and the number of repetitions completed. Similar findings were reported by Behrens et al. [50] who showed that caffeine consumption increased power performance of the plantar flexor muscles but did not increase MVC strength. One plausible reason may be that maximal and near-maximal squat performance is limited by the sticking region (area where bar velocity is minimal) located superior to the bottom parallel position close to an absolute thigh angle of approximately $30^{\circ}$ [51]. Surpassing the sticking region increases the likelihood of completing a successful repetition as bar velocity increases during the remainder of the range of motion until the deceleration phase ensues [51]. Although power and velocity were higher during each repetition, it is possible the greater power did not transfer through the sticking region when fatigue reached maximal levels. Nevertheless, the higher power and velocity values observed during the SCF treatment may be viewed as beneficial and could potentially lead to greater power gains during long-term training periods.

Average and peak concentric force decreased from set 1 through set 6 at various points. However, no supplement effect was observed with regard to the pattern of reduction during each set. Although S and SCF treatments yielded higher peak concentric force values (of up to $2.5 \%$ ) than CT and P, respectively, these differences did not reach statistical significance. These data demonstrate that the velocity increase was the main contributor to increased power seen in SCF. Although force and velocity are positively related, the small relative peak concentric force increases observed in SCF and S did not reach statistical significance. Nevertheless, this small mean difference appeared large enough to increase bar velocity and subsequent power performance.

The results of the present study showed a progressive increase in RPE during each successive set with no supplement effects observed. Numerous studies have examined the effects of caffeine on RPE. These studies have shown either no effect of caffeine on RPE [41-43, 46, 49] or a reduced RPE response [24, 40] during resistance exercise. Doherty and Smith [52] conducted a meta-analysis of studies examining caffeine effects on RPE during exercise and concluded that caffeine reduced RPE by $5.6 \%$ with a concomitant $11.2 \%$ increase in performance. RPE accounted for $29 \%$ of the variance in exercise performance [52]. Haller et al. [11] examined Ripped Fuel (21 mg of synephrine and $304 \mathrm{mg}$ of caffeine) during $30 \mathrm{~min}$ of cycling at 75-80\% $\mathrm{VO}_{2}$ max and reported lower RPE during exercise. Thus, our data support studies during resistance exercise showing similar RPE values between caffeine and placebo conditions. The results of the present study indicated that $p$-synephrine alone or in combination with caffeine yielded similar RPE during resistance exercise. Considering that significant performance improvements were seen in both S and SCF treatments, these data indicate that both supplements can blunt acute increases in RPE that would be expected with a greater volume load of resistance exercise.

A similar finding was observed in blood lactate. Significant elevations in blood lactate were seen at T3, T4, and T5 with no differences observed between treatments. Haller et al. [11] reported no difference in blood lactate response between Ripped Fuel and placebo treatments during cycling. Studies examining the effects of caffeine consumption on blood lactate response to resistance exercise have shown no differences [40] or greater blood lactate concentrations [24] as performance enhancement was shown. The results of the present study indicate that $100 \mathrm{mg}$ of $p$-synephrine increased repetition performance and volume load without increasing the blood lactate response. The addition of $100 \mathrm{mg}$ of caffeine did not augment the response. Thus, both the S and SCF supplements enhanced performance without eliciting a greater lactate response.

The results of the present study should be viewed within the design limitations. The supplementation protocol was based on using absolute dosing in lieu of relative dosing as the intent was to examine the supplements in their available chew form. In addition, subjects consumed the supplements for three days prior to performing each protocol. Thus, the acute resistance exercise response to a single dose remains to be seen. Future studies should address $p$-synephrine supplementation using a variety of doses for different types of resistance exercise programs and should examine potential chronic training effects. 


\section{Conclusions}

This study demonstrated that $100 \mathrm{mg}$ of $p$-synephrine alone or in combination with $100 \mathrm{mg}$ of caffeine significantly augmented resistance exercise repetition performance and volume load. The addition of caffeine to $p$-synephrine resulted in faster, more powerful repetitions. These changes took place without a concomitant increase in RPE or blood lactate indicating that $p$-synephrine (with and without caffeine) supplementation can blunt acute increases in RPE and blood lactate known to accompany a greater volume of exercise. The resultant effects are increased local muscular endurance and more powerful repetitions (when caffeine is added) with no additional perceived exertion or lactate accumulation.

\section{Competing interests}

SJS has served as a consultant to Nutratech, Inc. All other authors report no competing interests.

\section{Authors' contributions}

$N A R, J A B$, and JK were involved with study design, subject recruitment, scheduling and coordination, protocol setup and supervision, data acquisition, data analysis and interpretation, and preparation of the manuscript. SJS, WJK, and ADF were involved in study design, data interpretation, and preparation of the manuscript. VGN, MDL, and KBD were involved in subject scheduling and coordination, equipment calibration, protocol setup, data acquisition, data entry, and analysis. All authors read and approved the final manuscript.

\section{Acknowledgments}

We would like to thank a dedicated group of subjects for their participation in this study. We would like to thank Lauren Pigott, Joshua Pacifico, Cassandra Noonan, Ryan Kar, Victoria Davila, and Amber Schlosser for their assistance with data collection and analysis. This study was funded from a grant from Nutratech, Inc., West Caldwell, NJ.

\section{Author details}

Department of Health and Exercise Science, The College of New Jersey, Ewing, NJ 08628, USA. ${ }^{2}$ Department of Human Sciences, The Ohio State University, Columbus, $\mathrm{OH}$ 43210, USA. ${ }^{3}$ School of Pharmacy and Health Professions, Creighton University, Omaha, NE 68178, USA.

Received: 10 April 2015 Accepted: 2 September 2015

Published online: 17 September 2015

\section{References}

1. Stohs SJ, Preuss HG, Keith SC, Keith PL, Miller H, Kaats GR. Effects of $p$-synephrine alone and in combination with selected bioflavonoids on resting metabolism, blood pressure, heart rate and self-reported mood changes. Int J Med Sci. 2011;8:295-301.

2. Wilborn C, Taylor L, Poole C, Bushey B, Williams L, Foster C, et al. Effects of ingesting a commercial thermogenic product on hemodynamic function and energy expenditure at rest in males and females. Appl Physiol Nutr Metab. 2009;34:1073-8.

3. Hoffman JR, Kang J, Ratamess NA, Jennings PF, Mangine G, Faigenbaum $A D$. Thermogenic effect from nutritionally enriched coffee consumption. J Int Soc Sports Nutr. 2006:3:35-41.

4. Hoffman JR, Kang J, Ratamess NA, Rashti SL, Tranchina CP, Faigenbaum AD. Thermogenic effect of an acute ingestion of a weight loss supplement. J Int Soc Sports Nutr. 2009;6:1-9.

5. Rashti SL, Ratamess NA, Kang J, Faigenbaum AD, Chilakos A, Hoffman JR. Thermogenic effect of Meltdown RTD ${ }^{\text {TM }}$ energy supplement in young healthy women: a double blind, cross-over design. Lipids Health Dis. 2009;8:57.

6. Lopez HL, Ziegenfuss TN, Hofheins JE, Habowski SM, Arent SM, Weir JP, et al. Eight weeks of supplementation with a multi-ingredient weight loss product enhances body composition, reduces hip and waist girth, and increases energy levels in overweight men and women. J Int Soc Sports Nutr. 2013;10:22

7. Hoffman JR, Kang J, Ratamess NA, Jennings PF, Mangine GT, Faigenbaum AD. Effect of nutritionally enriched coffee consumption on aerobic and anaerobic exercise performance. J Strength Cond Res. 2007;21:456-9.

8. Gonzalez AM, Walsh AL, Ratamess NA, Kang J, Hoffman JR. Effect of a pre-workout energy supplement on acute multi-joint resistance exercise. J Sports Sci Med. 2011;10:261-6.

9. Kraemer WJ, Hatfield DL, Spiering BA, Vingren JL, Fragala MS, Ho JY, et al. Effects of a multi- nutrient supplement on exercise performance and hormonal responses to resistance exercise. Eur J Appl Physiol. 2007;101:637-46.

10. Hoffman JR, Kang J, Ratamess NA, Hoffman MW, Tranchina CP, Faigenbaum $A D$. Examination of a pre-exercise, high energy supplement on exercise performance. J Int Soc Sports Nutr. 2009;6:1-8.

11. Haller CA, Duan M, Jacob P, Benowitz N. Human pharmacology of a performance-enhancing dietary supplement under resting and exercise conditions. Br J Clin Pharmacol. 2008;65:833-40.

12. Stohs SJ, Preuss HG, Shara M. A review of the human clinical studies involving citrus aurantium (bitter orange) extract and its primary protoalkaloid p-synephrine. Int J Med Sci. 2012;9:527-38.

13. Kaats GR, Miller H, Preuss HG, Stohs SJ. A 60 day double-blind, placebo-controlled safety study involving Citrus aurantium (bitter orange) extract. Food Chem Toxicol. 2013;55:358-62

14. Stohs SJ, Preuss HG, Shara M. A review of the receptor-binding properties of p-synephrine as related to its pharmacological effects. Oxid Med Cell Longev. 2011;2011:1-9.

15. Carpene C, Galitzky J, Fontana E, Atgie C, Lafontan M, Berlan M. Selective activation of beta-3-adrenoceptors by octopamine; comparative studies in mammalian fat cells. Naunyn Schmiedebergs Arch Pharmacol. 1999;359:310-21.

16. Goldstein ER, Ziegenfuss T, Kalman D, Kreider R, Campbell B, Wilborn C, et al. International Society of Sports Nutrition position stand: caffeine and performance. J Int Soc Sports Nutr. 2010;7:5.

17. Laurent D, Schneider KE, Prusaczyk WK, Franklin C, Vogel SM, Krssak M, et al. Effects of caffeine on muscle glycogen utilization and the neuroendocrine axis during exercise. J Clin Endocrinol Metab. 2000;85:2170-5.

18. Davis JK, Green JM. Caffeine and anaerobic performance: ergogenic value and mechanisms of action. Sports Med. 2009;39:813-32.

19. Bazzucchi I, Felici F, Montini M, Figura F, Sacchetti M. Caffeine improves neuromuscular function during maximal dynamic exercise. Muscle Nerve. 2011:43:839-44.

20. Pesta DH, Angadi SS, Burtscher M, Roberts CK. The effects of caffeine, nicotine, ethanol, and tetrahydrocannabinol on exercise performance. Nutr Metabol. 2013;10:71

21. Jacobson BH, Weber MD, Claypool L, Hunt LE. Effect of caffeine on maximal strength and power in elite male athletes. Br J Sports Med. 1992;26:276-80.

22. Graham TE, Battram DS, Dela F, El-Sohemy A, Thong FS. Does caffeine alter muscle carbohydrate and fat metabolism during exercise? Appl Physiol Nutr Metab. 2008;33:1311-8.

23. Timmins TD, Saunders DH. Effect of caffeine ingestion on maximal voluntary contraction strength in upper- and lower-body muscle groups. J Strength Cond Res. 2014;28:3239-44.

24. Duncan MJ, Oxford SW. Acute caffeine ingestion enhances performance and dampens muscle pain following resistance exercise to failure. J Sports Med Phys Fit. 2012;52:280-5.

25. Hendrix CR, Housh TJ, Mielke M, Zuniga JM, Camic CL, Johnson GO, et al. Acute effects of a caffeine-containing supplement on bench press and leg extension strength and time to exhaustion during cycle ergometry. J Strength Cond Res. 2010;24:859-65.

26. Beck TW, Housh TJ, Malek MH, Mielke M, Hendrix R. The acute effects of a caffeine-containing supplement on bench press strength and time to running exhaustion. J Strength Cond Res. 2008;22:1654-8.

27. Warren GL, Park ND, Maresca RD, McKibans Kl, Mallard-Stafford ML. Effect of caffeine ingestion on muscular strength and endurance: a meta-analysis. Med Sci Sports Exerc. 2010;42:1375-87.

28. Juliano LM, Griffiths RR. A critical review of caffeine withdrawal: empirical validation of symptoms and signs, incidence, severity, and associated features. Psychopharmacology (Berl). 2004;176:1-29.

29. Jackson A, Pollock M. Generalized equations for predicting body density of men. Br J Nutr. 1978;40:497-504.

30. Siri WE. Gross composition of the body. In: Lawrence $J H$, Tobias CA, editors Advances in biological and medical physics, IV. New York: Academic; 1956. 
31. Kraemer WJ, Fry AC, Ratamess NA, French DN. Strength testing: development and evaluation of methodology. In: Maud P, Foster C, editors. Physiological assessment of human fitness. 2nd ed. Champaign: Human Kinetics; 2006. p. 119-50.

32. Faigenbaum AD, Ratamess NA, McFarland J, Kaczmarek J, Corragio MJ, Kang $J$, et al. Effect of rest interval length on bench press performance in boys, teens, and men. Ped Exerc Sci. 2008;20:457-69.

33. Peixoto JS, Comar JF, Moreira CT, Soares AA, de Oliveira AL, Bracht A, et al. Effects of Citrus aurantium (bitter orange) fruit extracts and p-synephrine on metabolic fluxes in the rat liver. Molecules. 2012;17:5854-69.

34. Hong NY, Cui ZG, Kang HK, Lee DH, Lee YK, Park DB. p-Synephrine stimulates glucose consumption via AMPK in L6 skeletal muscle cells. Biochem Biophys Res Commun. 2012;418:720-4.

35. Murphy $K T$, Bundgaard $H$, Clausen T. $\beta_{3}$-adrenoceptor agonist stimulation of the $\mathrm{Na}+, \mathrm{K}_{+}-$pump in rat skeletal muscle is mediated by $\beta_{2}$-rather than $\beta_{3}$-adrenoceptors. Br J Pharmacol. 2006;149:635-46.

36. Miniaci MC, Bucci M, Santamaria R, Irace C, Cantalupo A, Cirino G, et al. CL 316,243 , a selective $\beta 3$-adrenoceptor agonist, activates protein translation through mTOR/p7056K signaling pathway in rat skeletal muscle cells. Pflugers Arch. 2013;465:509-16.

37. Williams AD, Cribb PJ, Cooke MB, Hayes A. The effect of ephedra and caffeine on maximal strength and power in resistance-trained athletes. J Strength Cond Res. 2008;22:464-70.

38. Jacobs I, Pasternak H, Bell DG. Effects of ephedrine, caffeine, and their combination on muscular endurance. Med Sci Sports Exerc. 2003;35:987-94.

39. Duncan MJ, Thake CD, Downs PJ. Effect of caffeine ingestion on torque and muscle activity during resistance exercise in men. Muscle Nerve. 2014:50:523-7.

40. Duncan MJ, Stanley M, Parkhouse N, Cook K, Smith M. Acute caffeine ingestion enhances strength performance and reduces perceived exertion and muscle pain perception during resistance exercise. Eur J Sport Sci. 2013:13:392-9.

41. Hudson GM, Green JM, Bishop PA, Richardson MT. Effects of caffeine and aspirin on light resistance training performance, perceived exertion, and pain perception. J Strength Cond Res. 2008;22:1950-7.

42. Green JM, Wickwire PJ, McLester JR, Gendle S, Hudson G, Pritchett RC, et al. Effects of caffeine on repetitions to failure and ratings of perceived exertion during resistance training. Int J Sports Physiol Perform. 2007;2:250-9.

43. Beck TW, Housh TJ, Schmidt RJ, Johnson GO, Housh DJ, Coburn JW, et al. The acute effects of a caffeine-containing supplement on strength, muscular endurance, and anaerobic capabilities. J Strength Cond Res. 2006:20:506-10.

44. Mora-Rodriguez R, Garcia Pallares J, Lopez-Samanes A, Ortega JF, Fernandez-Elias VE. Caffeine ingestion reverses the circadian rhythm effects on neuromuscular performance in highly resistance-trained men. PLoS One. 2012;7:333807.

45. Trevino MA, Coburn JW, Brown LE, Judelson DA, Malek MH. Acute effects of caffeine on strength and muscle activation of the elbow flexors. J Strength Cond Res. 2015;29:513-20.

46. Astorino TA, Rohmann RL, Firth K. Effect of caffeine ingestion on one-repetition maximum muscular strength. Eur J Appl Physiol. 2008;102:127-32.

47. Astorino TA, Roberson DW. Efficacy of acute caffeine ingestion for shortterm high-intensity exercise performance: a systematic review. J Strength Cond Res. 2010;24:257-65.

48. Astorino TA, Martin BJ, Schachtsiek L, Wong K, Ng K. Minimal effect of acute caffeine ingestion on intense resistance training performance. J Strength Cond Res. 2011:25:1752-8.

49. Astorino TA, Terzi MN, Roberson DW, Burnett TR. Effect of two doses of caffeine on muscular function during isokinetic exercise. Med Sci Sports Exerc. 2010;42:2205-10.

50. Behrens M, Mau-Moeller A, Heise S, Skripitz R, Bader R, Bruhn S. Alteration in neuromuscular function of the plantar flexors following caffeine ingestion. Scand J Med Sci Sports. 2015;25:e50-8.

51. McLaughlin TM, Dillman CJ, Lardner TJ. A kinematic model of performance in the parallel squat by champion powerlifters. Med Sci Sports. 1977;9:128-33.

52. Doherty $M$, Smith PM. Effects of caffeine ingestion on rating of perceived exertion during and after exercise - a meta-analysis. Scand J Med Sci Sports. 2005;15:69-78.

\section{Submit your next manuscript to BioMed Central and take full advantage of:}

- Convenient online submission

- Thorough peer review

- No space constraints or color figure charges

- Immediate publication on acceptance

- Inclusion in PubMed, CAS, Scopus and Google Scholar

- Research which is freely available for redistribution

Submit your manuscript at www.biomedcentral.com/submit 